\title{
Microscopy Handbook
}

Describes the most common immunocytochemical methods in detail with explanatory diagrams and photomicrographs. Intended readership includes biomedical undergraduates, postgraduates, researchers and technicians.

Paperback, 72 pages, 8 line illus.; 10 half-tones, 4 tables. ISBN: 0198564155 Available from Microscopy Today at a price of $\$ 20.00$ plus $\$ 5.00$ S\&H (U.S.)

\section{Table of Contents}

1. Introduction

Definition

History and development

References

2. Production of Antibodies

Immunization

Testing

Region-specific antibodies

Monoclonal antibodies

Characteristics of a "good" antibody

References

3. Requirements for Immunocytochemistry Fixation

Cross-linking fixatives

Precipitant fixatives

Combination fixatives

Fresh tissue

Pre-fixed, non-embedded material

Freeze-drying

Tissue storage

Adherence of sections and cell

preparations to slides

Antigen retrieval in fixed tissues

Washing

Protease treatment

Heat-mediated antigen retrieval

Visualizing the end-product of reaction

Fluorescent labels

Enzyme labels

Colloidal gold

Other labels

Absence of non-specific staining

Causes and prevention of non-specific staining

Color Plates

4. Methods

General considerations

Buffer

Antibody diluent

Antibody dilution relative to reaction time temperature and technique

Methods

Nature of antibodies (IgG)

Application of antibodies to preparations

Direct method

Indirect method

Three-layer methods

Avidin-biotin methods

References

5. Specificity Problems and Essential Controls

Testing for non-specific binding due to tissue factors

Testing for non-specific binding by the primary antibody

Testing for non-specific binding of second and third reagents

Non-specific or unwanted specific staining due to antibody factors
Unwanted specific staining of unknown antigens

Non-specific binding of antisera to basic proteins in the tissue

Unwanted specific cross-reactivity of antiimmunoglobulin

Cross-reactivity of the primary antibody with related antigens

Remedies for non-specificity due to tissue factors Blocking binding sites with normal serum Absorption with tissue powder

Remedies for non-specificity due to heterogeneity of the antibody

Dilution

Affinity purification

Remedies for non-specifictiy due to cross-reactivity Controls

Negative controls

Positive control

Experimental contols

References

6. Enhancement of Standard Methods

Build-up methods

Intensification of the peroxidase/DAB $/ \mathrm{H}_{2} \mathrm{O}_{2}$ product Post-reaction intensification

Intensification during the peroxidase reaction Tyramine signal amplification(TSA)

References

7. Multiple Immunostaining

Primary antibodies raised in the same species Separately labelled primary antibodies Unlabelled primary, labelled secondary antibodies

Indirect double staining without elution

Double immunostaining with primary antibodies raised in different species

Triple immunostaining

References

8. Post-embedding Immunocytochemistry for the

Transmission Electon Microscope

Principles

Fixation

Processing to resin

Labels

Sectioning

Immunolabelling procedure

Pre-treatment

Immunolabelling

Contrasting

Multiple labelling

References

9. In Vitro Methods for Testing Antigen-Antibody

Reactions

Radioimmunoassay

Enzyme-linked immunosorbent assay (ELISA)

Western blotting

Dot blots

References
10. Applications of Immunocytochemistry Histopathological diagnosis Controls

Research

Quantification

Confocal microscopy

Flow cytometry and fluoresceny antibody

cell sorting (FACS)

Simpler methods of quantitation

Non-immunocytochemical uses of labelled probes

Receptor localization

Lectin histochemistry

In situ hybridization of nucleic acids References

11. Microscopy

References

Appendix: Technical Notes

Buffers

Phosphatase-buffered normal saline (PBS)

Tris-buffered normal saline (TBS)

Antibody diluent and storage of antibodies

Adherence of preparations to slides

Coating slides with poly-_ysine

Coating slides with silane

Blocking endogenous peroxidase reation

Paraffin sections

Milder methods for cryostat sections and

whole-cell preparations

Blocking endogenous biotin

Enzyme pre-treatment

Trypsin

Protease

Pepsin

Neuraminidase

Heat-mediated antigen retrieval using a

microwave oven

Enzyme development methods

Peroxidase

Alkaline phosphatase

Glucose oxidase

$\beta-0$-Galactosidase

Intensifying the peroxidase/DAB reaction product Following standard development

During development

Immunostaining methods

Initial procedure

Immunostaining - all preparations

Immunogold staining with silver enhancement

Silver acetate auto-metallography

Double immunoenyzmatic staining

Post-embedding electron microscopical

immunocytochemistry using epoxy resin-

embedded tissue and an indirect

immunogold method

Absorption specificity control (liquid phase)

References

Index 


\section{Seeing is believing The LEO 1500 series is here...}
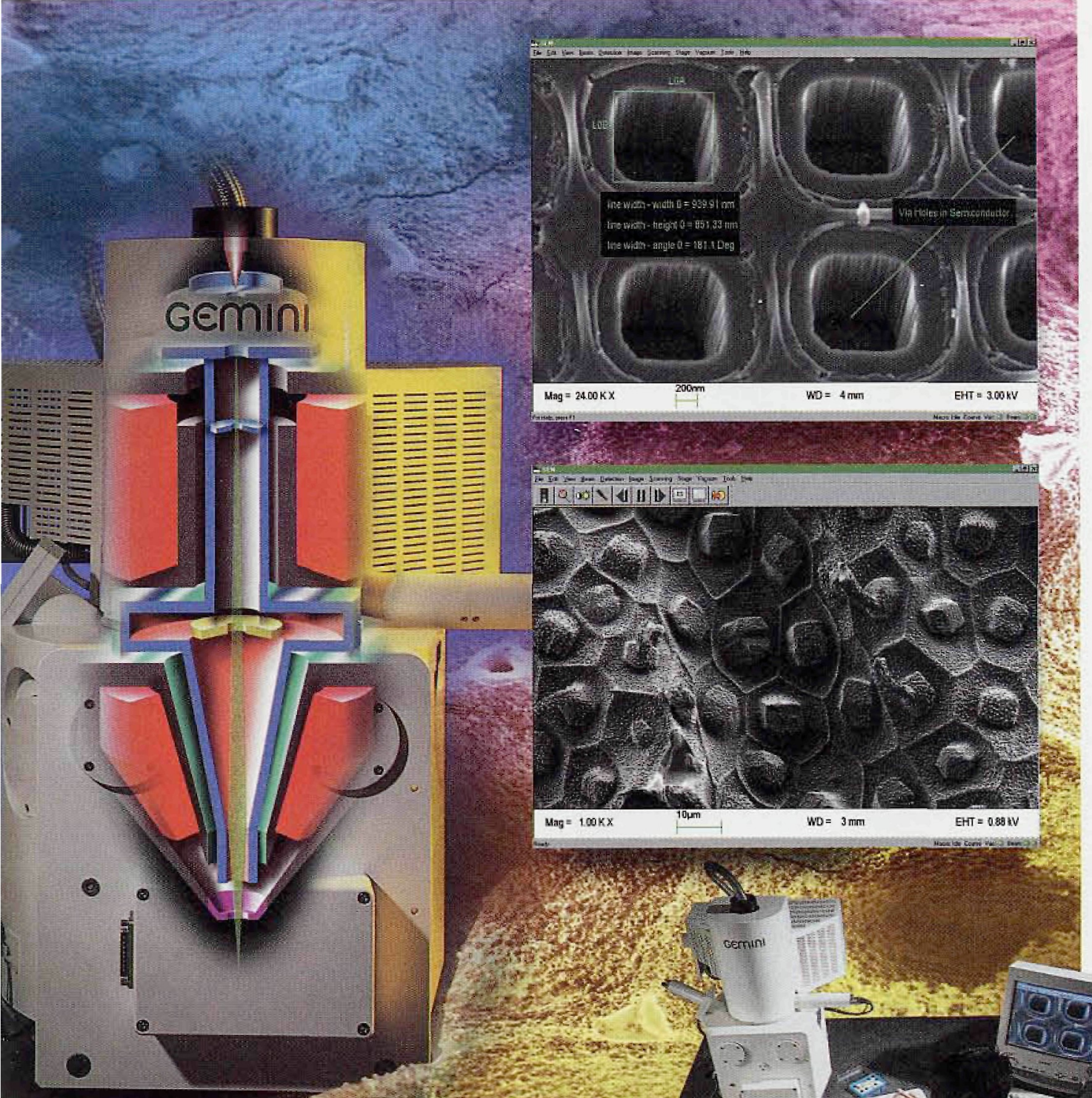

The LEO 1500 series, featuring the unique GEMINI electron optical column and a totally new control system has arrived. With a new generation of LEO's powerful software control running in Windows 95, the LE0 1500 series offers you the following powerful capabilities:

$\nabla$ Set up different users' access levels, with a log-on procedure using the built-in Administrator facility.

7 Save images to disk or network at pixel resolutions up to $3 k \times 2 k$, so that you can even zoom the magnification on stored images.

$\nabla$ Choose the beam voltage (200V to $30 \mathrm{kV}$ ) to suit the sample or the analysis, because the resolution is superb at any voltage.

7 Fast and accurate analysis with EDX, EBSP, $B S D$ thanks to the exceptionally stable high probe current.

$\nabla$ Produce hard copy results in a format that you need using any Windows ${ }^{\mathrm{T}}$ compatible printer - laser prints, colour dye subliminations, photographic etc.

Best of all, astonishing image quality is ot your fingertips. Coll your local LEO representative for information or a demonstration and you'll find that seeing really is believing.

LEO Electron Microscony

UK

Telephone (44) 1223414166 Fax (44) 1223412776

E-mail info@leo-em.co.uk

Website www.leo-em.co.uk Germany:

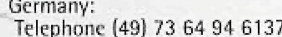
Telephone (49) 73649461
Fax (49) 7364944851 E-mail info@leode France:

Telephone (33) 141399210 Fax (33) 141399229 E-mail LEO_France@compuserve.com USA: Telephone (1) 9147477700 Fax (1) 9146817443 E-mail 70142.504@compuserve.com Plus a worldwide network of dealers

Visit us at the MSA Conference - Booth $210-215$

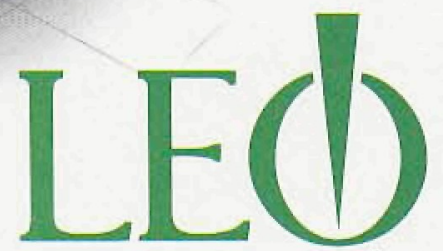




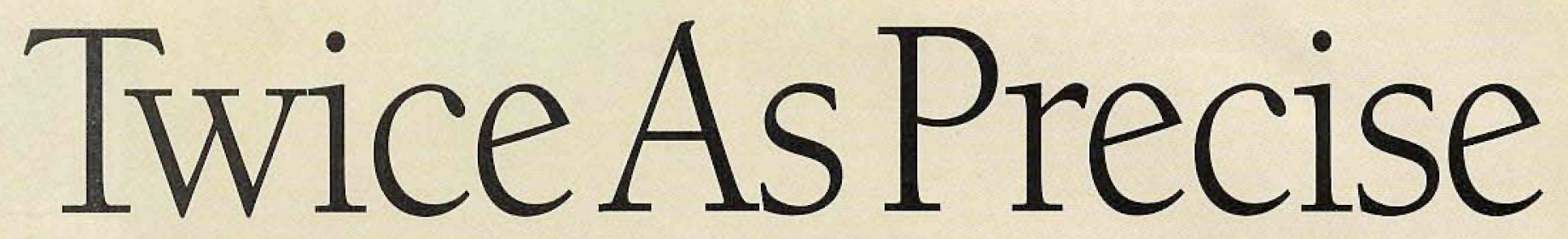

\section{JSM-5800 Scanning Microscope Features Two Options for Optimum Control.}

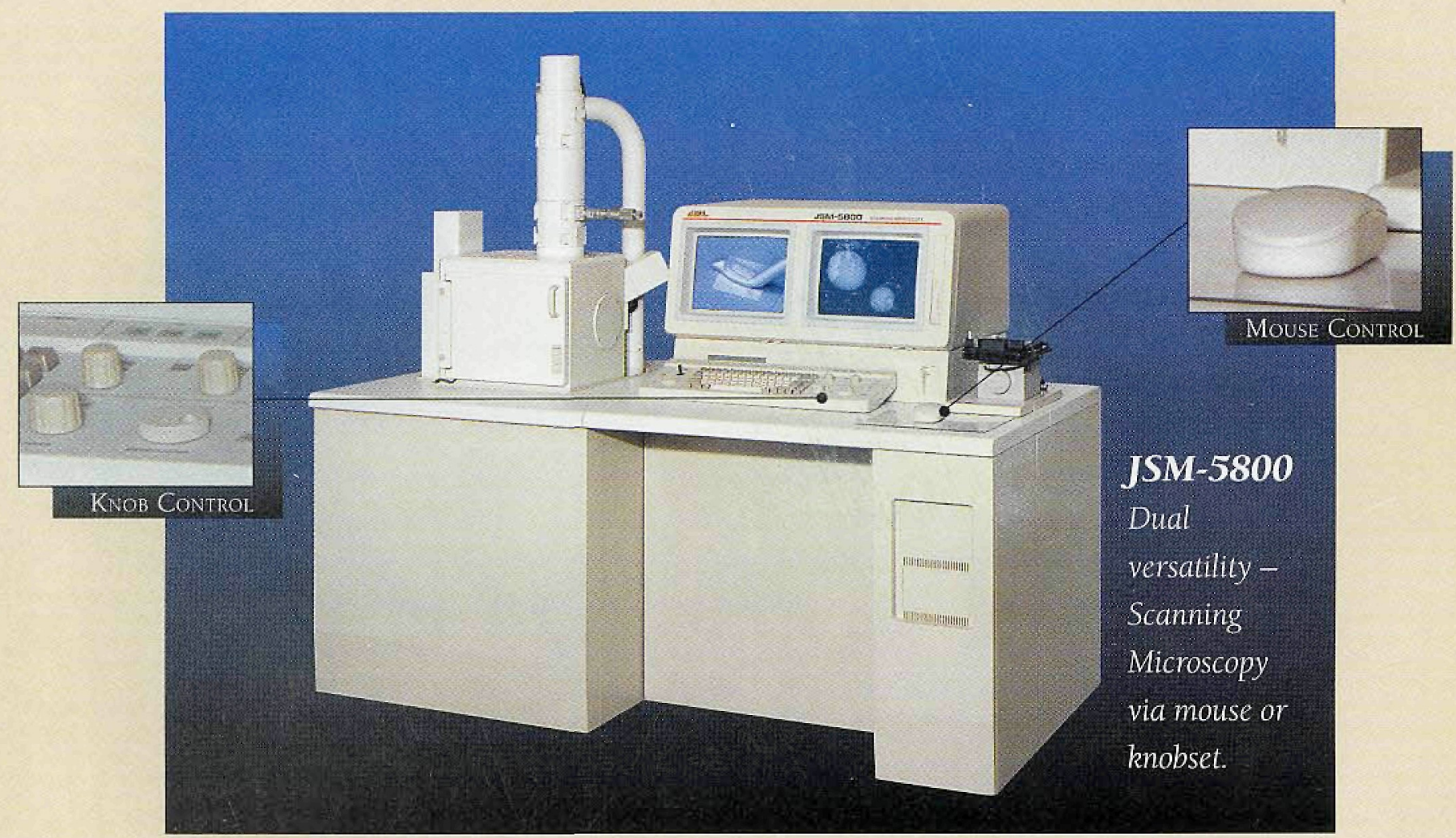

- Large Specimen Stage High/low vacuum capability

Suitable for a wide range of applications, the JSM-5800 from JEOL represents a new era in scanning microscopy. Now you have the option to choose either mouse or knobset control, while taking advantage of the super conical objective lens designed for the highest resolution ( $3.5 \mathrm{~nm}$ ) and large sample tilting.
Easy-to-use unit has a wide range of built-in automatic functions.

Darge specimen stage allows room for up to an 8-inch sample.

D Archiving enables temporary or permanent storage and retrieval in standard TIF format.

Dive axis stage automation makes the JSM-5800 fast and easy-to-use.
Discover the twice as precise alternative that is as unique as your work itself.

To arrange for a demonstration of the innovative JSM-5800 call JEOL today.

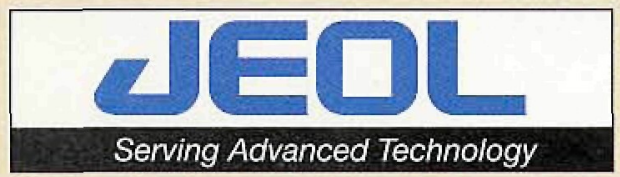

JEOL USA, Inc., 11 Dearborn Road, Peabody, MA 01960 Tel: 508-535-5900 Fax: 508-536-2205 e-mail: eod@jeol.com 